\title{
ABCD Spring Meeting Abstracts
}

\section{The ABCD Spring Meeting took place at Holywell Park Conference Centre, Loughborough on 16th \& 17th May 2019.}

\begin{abstract}
The authors of abstract 13 have requested that their abstract is not published.
\end{abstract}

\section{Prevalence of risk factors and burden of diabetic foot disease amongst patients on haemodialysis \\ Shah P, Connolly M, Knott A, Ledson T, Srinivas-Shankar U \\ Wirral University Teaching Hospital}

Aims: The aim of this study was to assess the prevalence of diabetes foot disease and its risk factors amongst patients undergoing haemodialysis.

Methods: This was a cross-sectional observational study of all patients with diabetes undergoing haemodialysis at a University Teaching Hospital. Case notes were reviewed for baseline characteristics, micro and macrovascular complications, past foot ulceration and amputation. Patients were examined for neuropathy, peripheral pulses, foot ulceration and foot deformity.

Results: 55/137 (40.1\%) patients receiving haemodialysis in hospital had diabetes. History and clinical examination was performed on 48 patients (mean (SD) age 64.3 (13.1) years). 26/48 (54.2\%) patients were male. Hypertension (89.6\%) and hyperlipidaemia $(70.8 \%)$ were the most frequent co-morbidities. $10.4 \%$ of patients reported symptoms of intermittent claudication, $16.2 \%$ of patients had venous eczema and $78.3 \%$ of patients had at least one palpable pulse. Neurotip perception was impaired in $55.2 \%$ and $50 \%$ of patients in the left and right foot, respectively. Vibration perception was impaired in $28.6 \%$ and $30.8 \%$ of patients at the left and right metatarsophalangeal joint area, respectively. Foot deformity was present in $38.5 \%$ of patients. $8(16.7 \%)$ patients had a previous amputation, $5(10.4 \%)$ had past Charcot neuroarthropathy and 9 (18.7\%) patients had a current foot ulcer.

Conclusions: There is a high prevalence of risk factors along with past and current foot disease among patients with diabetes on haemodialysis, highlighting the need for enhanced surveillance and early treatment.

Achieving glycaemic control in patients with diabetes on haemodialysis

Shah P, Connolly M, Knott A, Ledson T, Srinivas-Shankar U

Wirral University Teaching Hospital

Aim: Optimising glycaemic control in patients with end stage renal disease on haemodialysis can be a challenge. The aim of this study was to determine the treatment modalities in use to achieve optimal glycaemic control in these patients.

Methods: This was a cross-sectional observational study of patients with diabetes on haemodialysis at a University Teaching Hospital. Data collected included baseline characteristics, type and duration of diabetes, baseline biochemistry, treatment modality for diabetes and glycaemic control.
Results: 55/137 patients (40.1\%) undergoing haemodialysis had diabetes. Data were available for 48 patients (mean (SD) age 64.3 (13.1) years). 39 (81.3\%) patients had type 2 diabetes. Mean duration of diabetes was 19.6 (10.2) years. Body mass index was 29.3 (6.2), with $30(66.6 \%)$ patients being either overweight or obese. $\mathrm{HbA} 1 \mathrm{c}$ was 52.5 (17.6) $\mathrm{mmol} / \mathrm{mol}$ and serum creatinine was 560 (229) (normal range $59-104 \mu \mathrm{mol} / \mathrm{L}$ ). 14 patients $(29.2 \%$ ) were on diet-control alone. 25 patients (52.1\%) were on insulin and 13/25 $(46.4 \%)$ of these patients were on a basal bolus regimen. The total insulin dose was $36( \pm 24)$ units. Nine $(18.8 \%)$ patients were treated with oral hypoglycaemic agents (4 patients on sulfonylureas and 5 patients on dipeptidyl peptidase-4 inhibitors). Only two (4.2\%) patients were on both insulin and oral hypoglycaemic agents.

Conclusion: Despite the expanded armamentarium of therapeutic agents to treat diabetes, insulin remains the commonest treatment modality among patients with diabetes on haemodialysis.

Inpatient audit for recurrent diabetic ketoacidosis to highlight areas for service improvement in Warrington Hospital Rubab UR, Roua Abbas RA, Chattington PD, Ahmad MA Warrington and Halton Hospital

Objectives: A portion of patients with type 1 diabetes were repeatedly admitted for diabetes ketoacidosis (DKA) management. The audit aims to evaluate the precipitants and management of recurrent DKA in a district hospital in line with national guidelines, with a view to introducing measures to reduce the incidence.

Methods: A systemic review was performed of data from 218 patients hospitalised for DKA between 2017 and 2018 at our Trust.

Results: A total of 21 patients (9.6\%) were coded as recurrent DKA ( 2 or more episodes in year), male to female ratio $1: 1$, age 18-78 years (mean 42 years). Of the 21 cases with recurrent DKA, 14 $(67 \%)$ had true recurrent DKA. The other 7 were admitted for other reasons, which included non-ketotic hyperglycaemia and hyperosmolar hyperglycaemic state (HHS). For the 14 with recurrent DKA, $\mathrm{HbA} 1 \mathrm{c}$ nearest to the time of admission was $79-143 \mathrm{mmol} / \mathrm{mol}$, with a mean reading of $102 \mathrm{mmol} / \mathrm{mol}$. 1/14 was from prison, 1/14 from mental health unit, 5/14 $<25$ with 3-4 admissions per year. The main precipitant factor identified was infection in 9 patients (43\%); other factors included poor compliance (7/14) and eating disorders (2/14). When we looked at follow-up for these patients, only 3/14 patients were offered psychological support. All the patients had at least one post-DKA review with a diabetes specialist nurse (DSN). All had at least one telephone contact and many patients had frequent DSN support. 
Conclusion: We recognise that Warrington Diabetes Unit has the following services in place in line with the national guidelines:

1. Accredited structured education My Diabetes

2. Monday to Friday 08:00-18:00 helpline

3. Daily Diabetes in-reach team with 2 DSNs covering wards

4. Text to mobile before all clinics

5. Post DKA 1:1 education follow-up session plus ward support

6. Dual testing meter and ketone education to all

7. All DKA patients seen by DSN

8. Vulnerable group support

- Diabetes clinics are run in the local prison

- In-reach into the local psychiatric unit

- DSN teaching for residential and nursing home staff

- Sit in at anorexia clinic, but futile as it catches full-on anorexics and not diabulimia cases

Although we have been implementing national guidelines, our management is still suboptimal in some areas such as no out-of-hours service available, although there is advice through an algorithm on 111 , and no transition psychologist especially in providing psychological support for patients who have had more than one episode of DKA. We hope to have the service of a psychologist to work with patients having recurrent DKA as many of this group have complex needs.

Driving improvements in inpatient diabetes care: keep your eye on the dashboard!

Higgins K, Atkins, Oyayoyi D, Ball J

University Hospitals of Leicester NHS Trust, Leicester, UK

Background: In November 2017 the Care Quality Commission (CQC) issued a warning notice to our Trust raising concerns around the safe use of insulin. A robust action plan was implemented across all clinical directorates focusing on supporting frontline non-specialist staff. Assurance measures to track progress and a robust governance process were needed.

Aim: To develop an Inpatient Diabetes Dashboard to be shared quarterly at Executive Quality Board and with frontline staff.

Purpose: For assurance purposes, monitoring progress and to drive further change.

Method and results: With senior sponsors (nursing/medical), a business analyst, inpatient diabetes lead nurse and inpatient diabetes specialist pharmacist, we agreed measures to be reported in the dashboard. It was important to recognise limitations in data collection methods and to ensure the process was sustainable. We chose a combination of capillary blood glucose (CBG) data (\% CBG <3.0; $\%$ CBG >25.1). Data were collected via notes audit (medication errors) and datix incident reporting (National Inpatient Diabetes Harms audit). Compliance with insulin safety training was also reported. The dashboard was set up to report monthly from April 2018 and data are displayed on a single sheet in both tabular and graphical form.

Conclusion: The Leicester Inpatient Diabetes Dashboard provides a unique, visually appealing progress update which can be shared widely. It provides assurance within our organisation that we are addressing concerns raised by the CQC, national audit or local incident reporting. Importantly, it allows us to highlight where we need particular executive support to effect. The dashboard can be adapted for use within any acute trust.
Home diabetic foot ulcer service: a safe and clinically effective model for managing patients with diabetic foot ulcers in the community.

Wilson C, Coull P, Coggins C, Price L, Richards J, Ghaffar A

Dorset County Hospital NHS Foundation Trust. Williams Ave, Dorchester DT1 2JY, United Kingdom

Aims: In 2014-2015 the National Diabetes Foot Care Audit (NDFA) estimated that the cost of diabetic foot disease to the NHS in England is $\mathrm{f} 1$ billion per annum. ${ }^{1}$ We have introduced a service which aims to manage patients with diabetic foot ulcers in the community. This should result in financial gains and improved patient outcomes whilst in accordance with the national objective of managing patients in the community. Our 'Acute Hospital at Home' (AHAH) service is managed by a multidisciplinary team including medical consultants, junior doctors, microbiologists, nurses, physiotherapists and healthcare assistants.

Method: We have performed a retrospective analysis of 42 patients. 23 of these patients had their management aided by the AHAH service while 19 patients were solely treated in the hospital inpatient environment.

Results: The study shows that patients managed through AHAH had shorter length of inpatient stays ( 5.6 days vs 24.7 days). The cost of an AHAH bed is $f 100 /$ day and an acute medical bed is $f 280 /$ day, which results in a saving of $f 3,788$ per patient per admission from our study. Equivalence has been proved in rates of amputation, readmissions and death. We know that patient satisfaction is significantly better for those managed in the community. ${ }^{2}$

Conclusion: The results demonstrate clear benefits of managing patients with diabetic foot ulcers in the community - not only clear cost advantages but also significantly improved patient satisfaction and clinical outcome. We believe that the AHAH service is a safe and hugely valuable clinical service which could be widely applied across healthcare.

\section{References}

1. National Diabetes Foot Care Audit Hospital Admissions Report 2014-2016.

2. Cobb K, Jennison E, Graves J, et al: P59 Home bronchiectasis service: a safe and clinically effective model for managing infective exacerbations of bronchiectasis in the community. Thorax 2017;72:A113-A114.

Referral rates of patients with diabetes to secondary care are determined by practice size and confidence in treatment, not by $\mathrm{HbA} 1 \mathrm{c}$ level

Siah QZ, Ubeysekara NH, Davies S, Wong S, Dayan CM

School of Medicine, Cardiff University, Cardiff, UK; Centre of Diabetes and Endocrine Sciences, University Hospital of Wales, Cardiff, UK

Aims: To identify the factors affecting the referral rates of diabetic patients from primary care to secondary care.

Methods: A study was carried out based on 66 GP surgeries in the Cardiff and Vale University Health Board (population 515,581). $\mathrm{HbA} 1 \mathrm{c}$ outcome data of GP surgeries were obtained from the QOF database published for 2018. Referral rates were obtained from the electronic referral database of Cardiff and Vale University Health Board over the same period. Confidence level on the treatment of diabetes among GPs was assessed as a sub-study done in nine GP surgeries in the same area using a self-administered questionnaire. The relationship of adjusted referral rates with the GP practice size, $\mathrm{HbA} 1 \mathrm{c}$ outcome and the confidence level was assessed. 
Results: Average referral rates to secondary care in one year adjusted for number of patients with diabetes in each practice was $4.23 \%$, with a wide variation of $1.24 \%$ to $16.28 \%$. Average percentage of diabetic patients with $\mathrm{HbA} 1 \mathrm{c}$ level $<59 \mathrm{mmol} / \mathrm{mol}$ was $63.17 \%$ (range $43.19-76.23 \%$ ). The average confidence score of GPs in treating diabetes was 67\% (range 50-85\%) in the sub-study. The referral rate correlated inversely with the numbers of patients with diabetes in each practice $(p=0.008$; standardised correlation coefficient $=-0.332$ ), but there was no correlation with the $\mathrm{HbA} 1 \mathrm{c}$ outcome $(p=0.293$; standardised correlation coefficient $=-0.132)$. In a small sub-study, the referral rate appeared to correlate inversely with the confidence level of GPs in diabetes care.

Conclusions: Referral rates of patients with diabetes to secondary care are determined by practice size and possibly by confidence level in treatment, not by $\mathrm{HbA} 1 \mathrm{c}$ level. Further training in diabetes care for primary care teams may improve appropriateness of referral to secondary care.

Wide variation in the per patient cost of prescribing diabetes drugs for type 2 diabetes in primary care is not correlated with metabolic outcome

\section{Ubeysekara NH, Davies S, Wong S, Dayan CM}

Centre for Endocrine and Diabetes Sciences, University Hospital Wales, Cardiff; Cardiff and Vale University Health Board

Background: The prevalence of diabetes, especially type 2, is increasing all over the world. Diabetes prescribing has changed from older drugs to newer expensive drugs, causing an economic burden to health systems.

Aims: To identify the variation in prescription patterns and the cost of diabetes treatment across primary care practices in Cardiff and to compare expenditure versus outcome.

Methods: Data on 64 primary care institutions (515,581 patients) in Cardiff and Vale University Health Board area were obtained from Quality and Outcome Framework Wales 2018 database. Expenditure and volume of commonly used diabetes drugs were obtained from annually published government database (CASPA). Cost per patient for diabetes medication, prevalence of diabetes, prescription patterns and metabolic outcome (percentage of patients with $\mathrm{HbA} 1 \mathrm{C}$ $<58 \mathrm{mmol} / \mathrm{mol}$ ) were assessed and compared among 64 GP surgeries.

Results: Out of all patients, 4.94\% $(n=25,492)$ were diagnosed with diabetes. The rate of $\mathrm{HbA} 1 \mathrm{c}<58 \mathrm{mmol} / \mathrm{mol}$ was $63.17 \%$ (range $43-76 \%$ ). Biguanide usage was $43.6 \%$ of the total diabetes drug volume, but sulfonylurea usage was $15.2 \%$. Other drugs used included DPP-4 inhibitors (12.2\%), SGLT-2 inhibitors (8.8\%), GLP-1 agonists (3.0\%) and insulin (14.08\%). The average per patient cost for diabetes drugs was $£ 302.93$, with a wide range of $£ 207.00$ to f475.28. GP surgeries which reported lower expenditures used more biguanides and sulfonylureas than the newer drugs and the overall drug volume per patient was much less $(12.37,13.8$ vs $21.35,17.6)$. However, no relationship was seen between drugs used, cost per patient and the metabolic outcome measured by HbA1c.

Conclusions: There is a wide variation in prescribing patterns for patients with diabetes and annual per patient cost for diabetes among community GP surgeries which does not relate to improved metabolic outcomes. Substantial savings in prescribing costs for di- abetes drugs may be possible by retention of use of less expensive drugs in more patients without compromising metabolic outcomes.

\section{Aldosterone-renin ratio in screening for primary aldostero- nism: a clinical audit \\ Raheem AA, Cafferkey $M$ \\ Basildon University hospital, Nether Mayne, Basildon, SS16 5NL}

Background: The aldosterone-renin ratio (ARR) is the screening test of choice for primary aldosteronism. Patient selection and preparation are crucial to obtain meaningful results. We identified cases of inappropriate screening and significant numbers of repeated testing.

Aim: To compare current practice in a district general hospital against clinical guidelines and to identify the causes of repeated testing.

Methods: We searched the laboratory database for all ARR tests performed over a 14-month period from May 2016 to July 2017 in Basildon University Hospital. We reviewed the clinical records for indications for testing, and evaluated local practice against guidelines of the Endocrine Society in patients with hypertension and the European Society of Endocrinology in patients with adrenal nodules.

Results: We found 109 ARR measurements in 93 patients. $62 \%$ of patients were female. The indication for the test was hypertension in $52 \%$ and adrenal nodules in $46 \%$. In the hypertension group, $71 \%$ met the criteria for investigation while, in the adrenal nodule group, $40 \%$ of the patients met the criteria for screening. Overall, $59.1 \%$ met the criteria for investigation. Three patients were diagnosed with primary aldosteronism and another three cases are still under investigation. All positive results were in patients who met the criteria for screening. Interfering medications accounted for $33.3 \%$ of repeated tests.

Conclusion: ARR was not indicated in $40 \%$ of patients in this sample. Careful patient selection can reduce the numbers of unnecessary tests. A review of medications is necessary to avoid the need for repeat testing which is expensive and inconvenient for patients.

Improvement in renal function in the first UK NHS EndoBarrier service for uncontrolled diabesity

Ryder REJ, ${ }^{1}$ Sen Gupta $P_{1}^{1,2}$ Yadagiri $M,{ }^{1}$ Irwin $S P_{,}{ }^{1}$ Burbridge $W,{ }^{1}$ Bashir $T^{1}$ Allden RA, ${ }^{1}$ Bleasdale JP, Fogden $E N,{ }^{1}$ Anderson $M R,{ }^{1}$ Cockwell $P^{3}$

${ }^{1}$ City Hospital, Birmingham, UK; ${ }^{2}$ Guy's and St Thomas' Hospitals, London, UK; ${ }^{3}$ Queen Elizabeth Hospital, Birmingham, UK

Aims: EndoBarrier, a $60 \mathrm{~cm}$ proximal intestinal liner, endoscopically implanted for up to 1 year, reduces weight and $\mathrm{HbA} 1 \mathrm{c}$. As the risk of progressive kidney disease is increased by high body mass index (BMI), we assessed the impact of EndoBarrier on renal function.

Methods: Since October 2014 we have implanted 62 EndoBarriers in patients with suboptimally controlled diabesity in our NHS service and by November 2018 all were explanted. Outcomes were monitored in a registry.

Results: In 61 patients (mean \pm SD age $51.4 \pm 7.2$ years, $54.1 \%$ male, $57.4 \%$ Europid, diabetes duration 12.0 (8.0-19.5) years, $57.4 \%$ insulin-treated, BMl $41.9 \pm 7.4 \mathrm{~kg} / \mathrm{m} 2$ ) with implant and explant data, mean \pm SD $\mathrm{HbA} 1 \mathrm{c}$ fell by $23.7 \pm 21.4 \mathrm{mmol} / \mathrm{mol}$ from $80.2 \pm 22.5$ to 
$56.5 \pm 11.5 \mathrm{mmol} / \mathrm{mol}(p<0.001)$, weight fell by $15.9 \pm 8.5 \mathrm{~kg}$ from $122.6 \pm 27.9$ to $106.7 \pm 28.9 \mathrm{~kg}(p<0.001)$, systolic blood pressure (BP) fell from $138.5 \pm 15.0$ to $125.8 \pm 14.6 \mathrm{mmHg}(p<0.001)$, cholesterol fell from $4.7 \pm 1.4$ to $3.9 \pm 0.9 \mathrm{mmol} / \mathrm{L}(p<0.001)$ and alanineaminotransferase (ALT; liver fat marker) fell from $33.2 \pm 19.8$ to $19.5 \pm 11.4 \mathrm{U} / \mathrm{L}(\mathrm{p}<0.001)$. In the 35 insulin-treated patients the median (IQR) insulin dose was reduced from 100 (54-140) to 40 (0$70)$ units ( $p<0.001)$, with $10 / 35$ (28.6\%) discontinuing insulin. With regard to renal function, mean $\pm S D$ serum creatinine improved by $5.5 \pm 15.4 \mu \mathrm{mol} / \mathrm{L}$ from $91.7 \pm 47.7$ to $86.2 \pm 45.7 \mu \mathrm{mol} / \mathrm{L}(p=0.007)$ and estimated gomerular filtration rate (abbreviated MDRD equation) improved by $5.8 \pm 10.7 \mathrm{~mL} / \mathrm{min} / 1.73 \mathrm{~m} 2$ from $84.3 \pm 25.2$ to $90.1 \pm 26.4 \mathrm{~mL} / \mathrm{min} / 1.73 \mathrm{~m} 2(\mathrm{p}<0.001)$. Five patients had raised serum creatinine (>133 $\mu \mathrm{mol} / \mathrm{L})$ prior to Endobarrier; after implantation, in four of these creatinine was reduced and in two creatinine was normalised. It was noteworthy that the four patients with renal impairment who sustained improvement had large weight loss $(19.3-34.4 \mathrm{~kg})$ whereas the patient without improvement had only $6.6 \mathrm{~kg}$ weight loss.

Conclusion: As well as previously documented improvements in weight, HbA1c, BP, ALT and cholesterol, EndoBarrier was associated with improvements in renal function. These observational findings warrant further investigation.

Is electronic referral system an answer for better coordinated inpatient diabetes care?

Macey-Dare $A,{ }^{1}$ Zhang $Q,{ }^{1}$ Sim $N K,{ }^{2}$ Kaushal ${ }^{,}{ }^{2}$ Qureshi SA ${ }^{2}$

${ }^{1}$ Imperial College London, Medical School; ${ }^{2}$ Chelsea and Westminster NHS Foundation Trust, London, UK

Aims: Re-audit of the an electronic endocrine referral system to: (1) analyse the system's efficiency 1 year post-implementation; and (2) identify potential service gaps and service improvement areas.

Methods: A service level audit at a large district general hospital showed that a new electronic referral system dramatically improved timely access to specialist diabetes/endocrine advice. We re-audited the system by retrospectively analysing the referral audit trails from July to December 2018 (6 months).

Results: The primary outcome measure was the percentage of referrals responded to in 24 hours. On switching to electronic referrals, the proportion responded to within 24 hours rose from $25 \%$ in September 2016 to $82 \%$ in September 2017 and 76\% in September 2018. The figure for July to December 2018 was $76 \%$. Further analysis showed the majority of referrals (56.58\%) were responded to by diabetes specialist nurses (DSNs).

Conclusions: Our re-audit shows response times have remained similar, providing a strong case for continued use of this system as a superior alternative to paper referrals. The marginal decrease in the proportion of responses within 24 hours suggests a bottleneck at the point of referral authorisation as referral numbers have increased but workforce size has remained constant. This suggests a potential benefit to hiring more DSNs (as the main responders), and redistributing workload. The study was limited by its retrospective nature and by difficulties in comparing like-for-like time periods. Potential improvements include streamlining the "accept referral " function and making a "report" function available, so referral outcomes can be viewed easily online. All hospitals with electronic investigation-ordering systems could easily replicate our system.

\section{Diary dates 2020}

19-22 13th International Conference on

February Advanced Technologies \& Treatments for Diabetes (ATTD)

IFEMA Palacio Municipal,

Campo de las Naciones Avda. Capital de España Madrid 7-28042, Madrid, Spain

https://attd.kenes.com/

$\begin{array}{ll}17 & \text { YDEF Day } \\ \text { March } & \text { SEC Glasgow } \\ & \text { http://www.youngdiabetologists.org.uk/courses/ }\end{array}$

18-20 Diabetes UK Professional Conference 2020

March SEC Glasgow

https://www.diabetes.org.uk/Diabetes-UK-ProfessionalConference

$\begin{array}{ll}\text { 28-31 } & \text { ENDO } 2020 \\ \text { March } & \text { Moscone Center, San Francisco } \\ & \text { https://www.endocrine.org/endo2020 }\end{array}$

23-24 ABCD Spring Meeting 2020

April Hilton Liverpool City Centre

https://abcd.care/events/abcd-spring-meeting-2020

\begin{tabular}{|c|c|}
\hline $\begin{array}{l}23 \\
\text { April }\end{array}$ & $\begin{array}{l}\text { ABCD DTN-UK Meeting } \\
\text { Hilton Liverpool City Centre } \\
\text { https://abcd.care/events/abcd-dtn-uk-meeting-2020-0 }\end{array}$ \\
\hline $\begin{array}{l}27-29 \\
\text { April }\end{array}$ & $\begin{array}{l}\text { YDEF Technology Course } \\
\text { Contact Organizer for more detail: } \\
\text { info@youngdiabetologists.org.uk } \\
\text { http://www.youngdiabetologists.org.uk/courses/ }\end{array}$ \\
\hline $\begin{array}{l}13-15 \\
\text { May }\end{array}$ & $\begin{array}{l}\text { 18th Malvern Diabetic Foot Conference } \\
\text { Malvern Theatres, Grange Road WR14 3HB, } \\
\text { Worcestershire, United Kingdom } \\
\text { https://eu.eventscloud.com/ehome/mdfc2020/Home/ }\end{array}$ \\
\hline
\end{tabular}

23-26 European Congress of Endocrinology

May Prague, Czech Republic

https://www.ese-hormones.org/events-deadlines/europeancongress-of-endocrinology/ece-2020/

\begin{tabular}{ll}
\hline 12-16 & American Diabetes Association \\
June & 80th Scientific Session \\
& Chicago, Illinois \\
& https://professional.diabetes.org/scientific-sessions
\end{tabular}

\section{1-25 EASD 2020}

September 56th Annual Meeting of the European Association for the Study of Diabetes Vienna, Austria

https://www.easd.org/

For other meetings see https://www.abcd.care/events 